\title{
Gene polymorphisms in the ornithine decarboxylase-polyamine pathway modify gastric cancer risk by interaction with isoflavone concentrations
}

\author{
Lisa Y. Cho · Jae Jeong Yang $\cdot$ Kwang-Pil Ko $\cdot$ Seung Hyun Ma $\cdot$ Aesun Shin $\cdot$ Bo Youl Choi • \\ Hyun Ja Kim · Dong Soo Han · Kyu Sang Song · Yong Sung Kim · Soung-Hoon Chang • \\ Hai-Rim Shin $\cdot$ Daehee Kang $\cdot$ Keun-Young Yoo $\cdot$ Sue K. Park
}

Received: 30 August 2012/Accepted: 11 June 2014/Published online: 31 July 2014

(C) The International Gastric Cancer Association and The Japanese Gastric Cancer Association 2014

\begin{abstract}
Background The study aimed to examine the association between genes encoding molecules in the ornithine decarboxylase (ODC)-polyamine pathway (ODCl, AMDl, $N Q O 1, N O S 2 A$, and $O A Z 2$ ) and gastric cancer risk and whether the gene-phytoestrogen interaction modifies gastric cancer risk.

Methods Among 76 gastric cancer cases and their 1:4 matched controls within the Korean Multi-center Cancer Cohort, a total of 30 SNPs in five genes involved in the
\end{abstract}

Electronic supplementary material The online version of this article (doi:10.1007/s10120-014-0396-5) contains supplementary material, which is available to authorized users.

L. Y. Cho $\cdot$ J. J. Yang $\cdot$ S. H. Ma $\cdot$ D. Kang $\cdot$ K.-Y. Yoo $\cdot$

S. K. Park $(\square)$

Department of Preventive Medicine, Seoul National University

College of Medicine, 103 Daehakno, Jongno-Gu, Seoul 110-799,

Republic of Korea

e-mail: suepark@snu.ac.kr

J. J. Yang

e-mail: sweetj4u@snu.ac.kr

S. H. Ma

e-mail: justher@snu.ac.kr

D. Kang

e-mail: dhkang@snu.ac.kr

K.-Y. Yoo

e-mail: kyyoo@snu.ac.kr

L. Y. Cho · J. J. Yang - S. H. Ma - D. Kang · S. K. Park Cancer Research Institute, Seoul National University, Seoul, Korea

K.-P. Ko

Department of Preventive Medicine, Gachon University of Medicine and Science, Incheon, Korea

e-mail: kpko@gachon.ac.kr
ODC pathway were primarily analyzed. The second-stage genotyping in 388 matched case-control sets was conducted to reevaluate the significant SNPs interacting with phytoestrogens during the primary analysis. The summary odds ratios (ORs) [95\% confidence intervals (CIs)] for gastric cancer were estimated. Interaction effects between the SNPs and plasma concentrations of phytoestrogens (genistein, daidzein, equol, and enterolactone) were evaluated.

Results In the pooled analysis, NQO1 rs1800566 showed significant genetic effects on gastric cancer without heterogeneity [OR 0.83 (95\% CI 0.70-0.995)] and a greater decreased risk at high genistein/daidzein levels [OR 0.36 (95\% CI 0.15-0.90) and OR 0.26 (95\% CI 0.10-0.64),

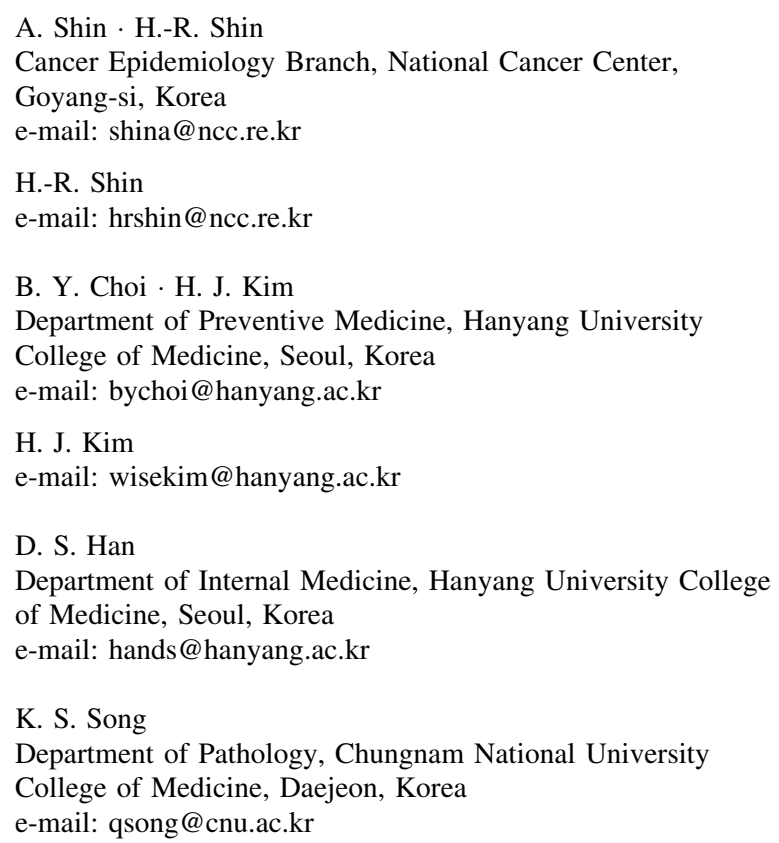


respectively; $\left.p_{\text {interaction }}<0.05\right]$. Risk alleles of $A M D 1$ rs1279599, AMD1 rs7768897, and OAZ2 rs7403751 had a significant gene-phytoestrogen (genistein and daidzein) interaction effect to modify the development of gastric cancer. They had an increased gastric cancer risk at low isoflavone levels, but a decreased risk at high isoflavone levels $\left(p_{\text {interaction }}<0.01\right)$.

Conclusions Our findings suggest that common variants in the genes involved in the ODC pathway may contribute to the risk of gastric cancer possibly by modulating ODC polyamine biosynthesis or by interaction between isoflavones and NQO1, OAZ2, and AMD1.

Keywords Gastric cancer - Ornithine decarboxylase . SNPs · Phytoestrogen · Polyamine $\cdot N Q O 1 \cdot O A Z 2 \cdot A M D 1$

\section{Introduction}

Helicobacter pylori is a major carcinogen for gastric cancer [1]. H. pylori infection results in inflammation and induces innate immunity in the host, which can be eradicated by host immune responses, such as nitric oxide (NO) production in macrophages. However, H. pylori-induced immune responses can fail to eradicate the bacterium by polyamine synthesis through the sequential ornithine decarboxylase (ODC)-polyamine pathway [12]. Elevated polyamine levels through the sequential ODC-polyamine pathway are associated with gastric cancer [2]. The ODCpolyamine pathway could contribute to gastric cancer by controlling chronic inflammation and host innate immune reaction, persistent $H$. pylori infection, and DNA damage (Fig. 1).

Since increased polyamine levels are associated with increased gastric cancer risk [2], decreases in polyamine levels may be key to reducing gastric cancer risk. Polyamine synthesis is stimulated by ODC, a rate-limiting

\section{Y. S. Kim}

Medical Genomics Research Center, Korea Research Institute of Bioscience and Biotechnology, Daejeon, Korea

e-mail: yongsung@kribb.re.kr

\section{S.-H. Chang}

Department of Preventive Medicine, Konkuk University, Chungju, Korea

e-mail: schang@kku.ac.kr

\section{H.-R. Shin}

Non Communicable Diseases and Health Promotion, World Health Organization, Western Pacific Regional Office, Manila, Philippines

D. Kang $\cdot$ S. K. Park

Department of Biomedical Sciences, Seoul National University Graduate School, Seoul, Korea enzyme, and $S$-adenosylmethionine decarboxylase (AMD), another key enzyme [3, 4], and thus inhibition of ODC and AMD may be of major importance. Previous studies showed that the irreversible inhibition of ODC suppressed cancer formation [5, 6]. Therefore, changes in these enzymes and dysfunction of homeostasis to control ODC and polyamine synthesis could be of paramount importance in gastric carcinogenesis. The sequential processes in the ODC-polyamine pathway can be regulated by their host genes, and therefore, it has been hypothesized that polymorphic variants of genes in the ODC-polyamine pathway may affect cancer susceptibility by altering their encoded enzymes, through either expression or function, to modulate polyamine synthesis.

Isoflavones have also been shown to affect ODC. The isoflavone genistein participates directly in inhibition of ODC activity and decreases polyamine levels [7]. It has been suggested that the interaction between genetic variants of enzymes in the ODC-polyamine synthesis pathway and isoflavone might be involved in modifying gastric cancer risk.

The objective of this study is twofold. First, the role of gene polymorphisms related to the ODC-polyamine pathway in the development of gastric carcinogenesis was investigated through a two-phase study that included (1) a discovery phase, which was a candidate gene approach focusing on five genes involved in the ODC pathway $(O A Z 2$, $O D C 1, A M D 1, N Q O 1$, and NOS2A) and (2) an extension phase, which further examined the significant singlenucleotide polymorphisms (SNPs) identified in the discovery genetic and interaction analysis. Second, the risk modification by gene-environment interaction between gene polymorphisms in the ODC-polyamine pathway and host serum phytoestrogen levels for gastric cancer was examined.

\section{Materials and methods}

\section{Ethics statement}

All participants signed an informed consent form before entering the studies. The study protocols for the Korean Multi-center Cancer Cohort (KMCC) study and the current nested case-control study were approved by the institutional review boards of Seoul National University Hospital and the National Cancer Center of Korea (H-0110-084002, C-0907-044-2861-170), and Hanyang University Hospital (2003-2004).

Study population

In the discovery phase, the population-based nested casecontrol study population was recruited from the KMCC, a 


\section{Modification in Gastric Cancer Risk}

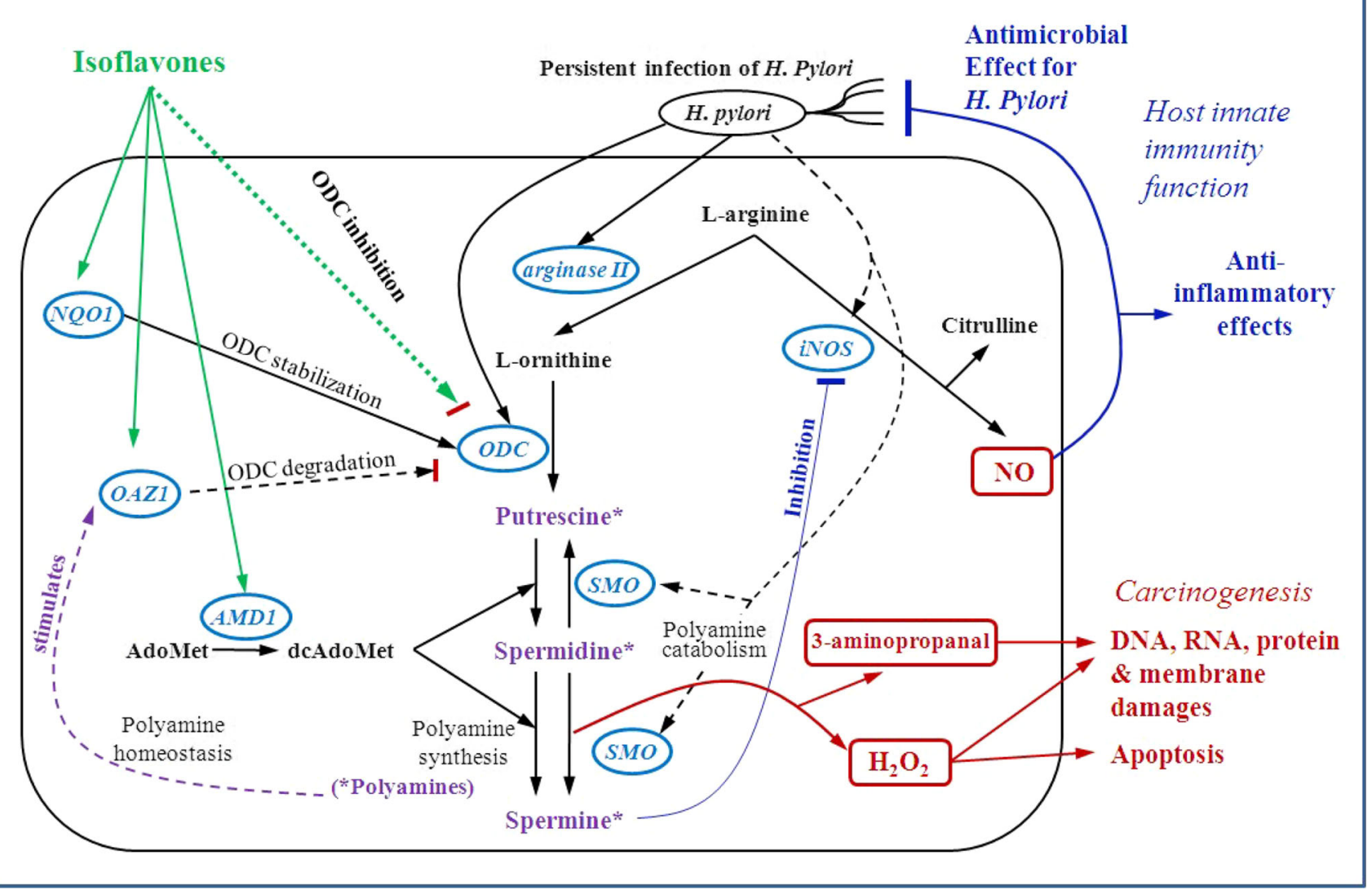

Fig. 1 The ornithine decarboxylase $(O D C)$-polyamine biosynthesis pathway. AdoMet $S$-adenosylmethionine, AMD1 $S$-adenosylmethionine decarboxylase 1, dcAdoMet decarboxylated $S$-adenosylmethionine,

community-based prospective cohort of participants recruited from four urban and rural areas in Korea (Haman, Chungju, Uljin, and Youngil) from 1993 through 2004 [8]. Participants signed informed consent forms and completed a detailed standardized interview-based questionnaire on general lifestyle, medical history, physical activity, diet, reproductive factors, pesticide exposure, and additional environmental factors. Blood and spot urine samples were collected and stored at -70 and $-20{ }^{\circ} \mathrm{C}$, respectively.

On December 31, 2002, 136 gastric cancer cases in the KMCC were identified through computerized record linkages to the national cancer registry, national death certificates, and health insurance medical records. The passive follow-up methods were reported to be $99 \%$ efficient, and completeness was assured [9]. Case patients diagnosed before recruitment $(N=36)$ and without blood samples $(N=16)$ were excluded. Cancer-free controls were randomly selected from the KMCC population. Four controls were matched to each gastric cancer case by incidence density sampling on the basis of age ( \pm 5 years), sex, residential district, and enrollment. Additionally, eight cases and 14 controls were excluded owing to insufficient DNA
iNOS inducible nitric oxide synthase, $N Q O 1 \mathrm{NAD}(\mathrm{P}) \mathrm{H}$ dehydrogenase, quinone $1, O A Z 1$ ornithine decarboxylase antizyme 1, SMO spermine oxidase

or poor genotyping. Finally, 76 cases and 322 controls were included in the discovery phase.

In the extension phase, 388 gastric cancer case-control sets were selected as follows. There were 95 new gastric cancer cases and 52 prevalent cases in December 2008 and 52 additional case patients whose blood samples were later obtained from the KMCC. In addition, from March 2002 to September 2006, 490 newly diagnosed gastric cancer cases from two university hospitals in Korea (Chungnam University Hospital and Hanyang University GURI Hospital) were identified. Epidemiological data and venous blood samples were collected at the time of diagnosis or prior to gastric cancer surgery. Of the newly diagnosed gastric cancer cases, 189 patients with sufficient DNA samples and who had provided informed consent were included. Community-based controls matched by age ( \pm 5 years), sex, and enrollment year from 2001 to 2005 were randomly selected from the KMCC. Two cases and 40 controls were excluded owing to poor genotyping and insufficient sample. Finally, 386 cases and 348 controls were included in the extension phase. Pooled analysis and meta-analysis included 462 cases and 670 controls. 


\section{Candidate gene and SNP selection}

In the discovery phase, five genes involved in the ODC pathway were selected as follows: $O D C 1$, which encodes OCD1, AMD1, which encodes AMD1; OAZ2, which encodes ornithine decarboxylase antizyme 2; NQO1, which encodes NAD $(\mathrm{P}) \mathrm{H}$ dehydrogenase, quinone 1 (NQO1); and NOS $2 A$, which encodes inducible nitric oxide synthase (iNOS).

SNPs from the genes were selected according to the following criteria: (1) SNPs that were reported to have a possible functional significance in previous studies; (2) SNPs with a minor allele frequency greater than 0.05 in Asian databases such as SNP500Cancer and CGAP using dbSNP IDs (http://www.ncbi.nlm.nih.gov/SNP); (3) concurrently, SNPs with a minor allele frequency greater than 0.05 in HapMap Japanese (JPT). Finally, 30 SNPs with a design score of $1.1, r^{2}>0.8$, were selected: 20 SNPs located in the intron region, seven SNPs located in the promoter region, and three SNPs located in the coding region.

In the extension phase, SNPs were selected as follows. In the discovery analysis of the single SNP association, no SNPs were found to be significant. In the gene-environment interaction analysis, NQO1 rs1800566 and rs1437135, OAZ1 rs7403751, and AMD1 rs1279599, rs7768897, and rs811921 were selected owing to a significant $p$ value for interaction $(p<0.05)$ and a lower raw $p$ value $(p<0.05)$ at low or high phytoestrogen level by classification of the median. Of the three AMD1 SNPs and four NQO1 SNPs, tagging SNPs such as rs1279599 and rs7768897 in AMD1 and rs740375 in NQO1 were selected. For $O A Z 2$, rs1800566 was selected.

\section{Genotyping}

Genotyping in the discovery phase was performed in 20 using the GoldenGate ${ }^{\mathrm{TM}}$ assay (Illumina ${ }^{\circledR}$, San Diego, CA, USA). Of the 30 SNPs in the ODC pathway, five SNPs were deemed unusable owing to failure of genotyping (rs7208775, rs1137933) and a SNP call rate below $90 \%$ (rs6494486, rs2872753, rs2248814) and were excluded from the analysis. Finally, we analyzed 25 SNPs in five genes in the ODC pathway (genotyping rate of $99.5 \%$ ). To ensure quality control and evaluate the intrasubject concordance rate, 52 duplicate samples were randomly distributed in the genotyping plate. Concordance rates for all assays were greater than $99 \%$.

Genotyping in the extension phase was performed in 20 using the Illumina VeraCode GoldenGate assay with BeadXpress according to the manufacturer's protocol (Illumina ${ }^{\circledR}$, San Diego, CA, USA) [16]. To ensure the reliability of the two different genotyping methods, 135 samples (59 cases and 76 controls) were genotyped twice by both the genome-wide human SNP Array 5.0 and the Illumina VeraCode GoldenGate assay, and the concordance rate was greater than $98.2 \%$. DNA concentration was determined using a NanoDrop ND-1000 spectrophotometer for the primary quality controls.

\section{Measurement of phytoestrogen biomarkers}

Plasma concentrations of four phytoestrogen biomarkersgenistein, daidzein, and equol (isoflavones) and enterolactone (a lignan) - were measured using time-resolved fluoroimmunoassay kits (Labmaster, Finland) in 2010. Free phytoestrogen biomarkers were extracted from $200 \mu \mathrm{L}$ of plasma, and the time-resolved fluorescence was measured using a VICTOR3 ${ }^{\text {TM }} 1420$ multilabel counter (PerkinElmer). Full details of the measurement method are described elsewhere [10]. Among the total genetic study population, the phytoestrogen concentrations of 406 case patients and 417 controls were measured, and these were analyzed in the gene-environment interaction analysis.

\section{Statistical analysis}

$\chi^{2}$ and Student $t$ tests were conducted to compare selected characteristics between gastric cancer cases and controls. Differences in selected characteristics of sex, age, $H$. pylori infection, CagA and VacA seropositivity, cigarette smoking, alcohol drinking, and gastritis history between cases and controls were determined by $p=0.05$.

Hardy-Weinberg equilibrium (HWE) in the control group was evaluated using the $\chi^{2}$ test or Fisher's exact test with a cut-off level of HWE $<0.0001$. In the discovery analysis, the association between individual SNPs and gastric cancer risk was evaluated on the basis of raw and permutated $p$ values using the likelihood ratio test with one degree of freedom in the additive, dominant, and recessive models. The additive model assumes a dose-response effect with an increasing number of variant alleles. The dominant and recessive models are tests for the minor allele. If $d$ is the minor allele and $\mathrm{D}$ is the major allele, the dominant model is $\mathrm{DD}$ vs dd + Dd and the recessive model is dd vs DD + Dd. Permutated $p$ values were estimated by 100,000 permutation tests. Gastric cancer risk was calculated as odds ratios (ORs) and $95 \%$ confidence intervals (CIs) using an unconditional logistic regression model with adjustment for potential risk factors of age, smoking status (ever vs never), H. pylori infection (positive vs negative), and CagA seropositivity (positive vs negative) in the additive, dominant, and recessive models. Haploblocks were created and tagSNPs were identified in haplotype analysis.

In the extension phase, the most significant SNPs in the discovery phase were reanalyzed. On the basis of the 
additive and/or recessive models, gastric cancer risk was estimated as ORs and $95 \%$ CIs using an unconditional logistic regression model with adjustment for the aforementioned risk factors. Stratified analysis by high and low levels of phytoestrogen biomarkers where the cut-off levels were determined by the median level of controls in the discovery phase and splicing analysis in the pooled analysis was conducted using unconditional logistic regression models. Recessive models of the SNPs that have the most significant effect on gastric cancer risk were computed as ORs (95\% CIs) adjusted for the same covariates.

All statistical analyses were performed using SAS version 9.1 (SAS Institute, Cary, NC, USA), PLINK version 1.06 (http://pngu.mgh.harvard.edu/purcell/plink) [11], and Haploview 4.1 (http:www.broadinstitute.org/haploview/ haploview).

\section{Results}

There was no significant difference in the selected characteristics between cases and controls in the discovery and extension phases $(p>0.05)$. In the total population, a significantly greater number of case patients were seropositive for CagA and VacA (Table 1).

In the single SNP analysis for genes involved in the ODC pathway and gastric cancer in the discovery phase, there were no significant SNPs associated with gastric cancer $(p>0.05)$ (Table S1). Four phytoestrogen biomarkers were measured in a total of 823 subjects (406 cases and 417 controls). The overall serum concentrations of genistein, daidzein, and enterolactone in case patients were significantly lower than in controls $(p<0.001)$, whereas the serum concentration of equol did not reach statistical significance $(p=0.0977)$ (Table S2).

Table 2 shows minor allele frequencies in the discovery phase, extension phase, and pooled analyses and gastric cancer risk of four SNPs in three genes in the ODC pathway that were reanalyzed in the extension phase. The minor allele frequencies were similar in the discovery and extension phases. NQO1 rs1800566 was found to be significantly associated with a decreased risk of gastric cancer in the pooled analysis and meta-analysis [OR 0.83 (95\% CI 0.70-0.995), $p=0.047$, and OR $0.83(95 \%$ CI $0.69-1.00), p=0.049$, respectively] (Table 2).

The gene-environment interaction analysis in the discovery phase showed an interaction effect between SNPs in the ODC pathway and phytoestrogens. We found that $p_{\text {interaction }}$ was significant for the following SNPs and phytoestrogens: genistein/daidzein and NQO1 rs1800566; genistein/daidzein and NQO1 rs1437135; daidzein/enterolactone and $A M D 1$ rs1279599; daidzein/enterolactone and AMD1 rs7768897; daidzein/enterolactone and AMD1 rs811921; equol and $O A Z 2$ rs7403751 (Table 3).

Table 4 shows gene-environment interactions between four SNPs in three genes in the ODC pathway that were reanalyzed in the extension phase and four phytoestrogen concentrations. In the gene-environment pooled analysis, NQO1 rs1800566 significantly interacted with genistein and daidzein to modify gastric cancer risk

Table 1 Selected characteristics of gastric cancer cases and controls in the gene-environment interaction analysis

\begin{tabular}{|c|c|c|c|c|c|c|c|}
\hline & \multicolumn{2}{|c|}{ Discovery phase $\mathrm{a}^{\mathrm{a}, \mathrm{f}}$} & \multicolumn{2}{|c|}{ Extension phase $\mathrm{p}^{\mathrm{b}, \mathrm{f}}$} & \multicolumn{3}{|l|}{ Total } \\
\hline & $\begin{array}{l}\text { Cases } \\
(n=76) \\
N(\%)\end{array}$ & $\begin{array}{l}\text { Controls } \\
(n=322) \\
N(\%)\end{array}$ & $\begin{array}{l}\text { Cases } \\
(n=386) \\
N(\%)\end{array}$ & $\begin{array}{l}\text { Controls } \\
(n=348) \\
N(\%)\end{array}$ & $\begin{array}{l}\text { Cases } \\
(n=462) \\
N(\%)\end{array}$ & $\begin{array}{l}\text { Controls } \\
(n=670) \\
N(\%)\end{array}$ & $p$ \\
\hline $\mathrm{Age}^{\mathrm{c}}$ & $64.5(8.6)$ & $62.8(8.4)$ & $61.5(10.5)$ & $63.1(8.4)$ & $62.0(10.3)$ & $63.0(8.4)$ & 0.18 \\
\hline Female & $20(26.3)$ & $98(30.4)$ & $130(33.7)$ & $110(31.6)$ & $150(32.5)$ & $208(31.0)$ & 0.91 \\
\hline Helicobacter pylori infection positive & $64(84.2)$ & $271(84.2)$ & $342(88.6)$ & $299(85.9)$ & $406(87.9)$ & $570(85.0)$ & 0.38 \\
\hline CagA positive & $65(85.5)$ & $273(84.8)$ & $355(92.0)$ & $308(88.5)$ & $420(90.9)$ & $581(86.7)$ & 0.06 \\
\hline VacA postiive & $44(57.9)$ & $171(53.1)$ & $271(70.2)$ & $233(67.0)$ & $315(68.2)$ & $404(60.6)$ & 0.01 \\
\hline Ever smoker ${ }^{\mathrm{d}}$ & $52(68.4)$ & $183(56.8)$ & 238 (61.7) & $191(54.9)$ & $290(62.8)$ & 374 (55.8) & 0.11 \\
\hline Ever drinker ${ }^{\mathrm{e}}$ & $46(60.5)$ & $184(57.1)$ & $239(62.1)$ & $206(59.2)$ & $285(61.8)$ & $390(58.2)$ & 0.20 \\
\hline Gastric ulcer history & $8(10.5)$ & $25(7.8)$ & 59 (17.6) & $50(18.7)$ & $67(17.8)$ & $75(16.8)$ & 0.51 \\
\hline \multicolumn{8}{|c|}{${ }^{a}$ Merged data from genetic study results from the discovery phase and phytoestrogen measurement experiments conducted in 2008} \\
\hline \multicolumn{8}{|c|}{$\begin{array}{l}\text { b Merged data from genetic study results from the discovery and extension phases and phytoestrogen measurement experiments conducted in } \\
2008 \text { and } 2011\end{array}$} \\
\hline \multirow{2}{*}{\multicolumn{8}{|c|}{$\begin{array}{l}\text { c Mean age in years; median age for all cases and controls was } 62.9 \text { years. The age ranged from } 29 \text { to } 85 \text { years. } \\
\text { d Ever smoker defined as former and current smoker }\end{array}$}} \\
\hline & & & & & & & \\
\hline \multicolumn{2}{|c|}{ e Ever drinker defined as former and current drinker } & & & & & & \\
\hline 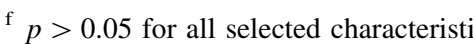 & & & & & & & \\
\hline
\end{tabular}


Table 2 Association between gastric cancer risk and single-nucleotide polymorphisms $(S N P)$ of genes involved in the ornithine decarboxylase (ODC) pathway in the pooled analysis and the meta-analysis

\begin{tabular}{|c|c|c|c|c|c|c|c|c|}
\hline \multirow[t]{2}{*}{ Gene } & \multirow[t]{2}{*}{ Chromosome } & \multirow[t]{2}{*}{ SNP } & \multirow[t]{2}{*}{ Genotype } & \multicolumn{3}{|c|}{ Genotype frequency } & \multicolumn{2}{|l|}{ OR $(95 \% \mathrm{CI})$} \\
\hline & & & & $\begin{array}{l}\text { Discovery } \\
\text { phase }\end{array}$ & $\begin{array}{l}\text { Extension } \\
\text { phase }\end{array}$ & $\begin{array}{l}\text { Pooled } \\
\text { analysis }\end{array}$ & Pooled analysis ${ }^{\mathrm{a}}$ & Meta-analysis $^{\mathrm{b}}$ \\
\hline \multirow[t]{4}{*}{$A M D 1$} & 6 & rs1279599 & $\mathrm{TT}$ vs $\mathrm{CC}+\mathrm{TC}$ & 0.20 & 0.20 & 0.20 & 1.0 & 1.0 \\
\hline & & & & 0.80 & 0.80 & 0.80 & $1.01(0.84-1.22)$ & $1.00(0.82-1.22)$ \\
\hline & & rs7768897 & $\mathrm{GG}$ vs $\mathrm{AA}+\mathrm{GA}$ & 0.21 & 0.20 & 0.21 & 1.0 & 1.0 \\
\hline & & & & 0.79 & 0.80 & 0.80 & $1.01(0.84-1.22)$ & $1.02(0.84-1.25)$ \\
\hline \multirow[t]{2}{*}{ OAZ2 } & 15 & rs7403751 & $\mathrm{CC}$ vs $\mathrm{GG}+\mathrm{CG}$ & 0.51 & 0.50 & 0.50 & 1.0 & 1.0 \\
\hline & & & & 0.49 & 0.50 & 0.50 & $1.12(0.83-1.52)$ & $1.12(0.81-1.54)$ \\
\hline \multirow[t]{2}{*}{$N Q O 1$} & 16 & rs 1800566 & $\mathrm{CC}$ vs $\mathrm{TT}+\mathrm{CT}$ & 0.13 & 0.13 & 0.13 & 1.0 & 1.0 \\
\hline & & & & 0.87 & 0.87 & 0.87 & $0.83(0.70-0.995)$ & $0.83(0.69-1.00)$ \\
\hline
\end{tabular}

Bold values indicate $p<0.05$

$C I$ confidence interval, $O R$ odds ratio

a Adjusted for age, smoking status, CagA seropositivity, and $H$. pylori in the dominant model.

${ }^{c}$ No heterogeneity (Cochran $\mathrm{Q}$ test, $p_{\text {heterogeneity }}>0.05$ )

Table 3 Selected SNPs of the genes involved in the ODC pathway interacting with phytoestrogens to modify gastric cancer risk in 67 gastric cancer cases and 209 controls (discovery scan)

\begin{tabular}{|c|c|c|c|c|c|c|c|c|}
\hline \multirow[t]{2}{*}{ Phytoestrogen } & \multirow[t]{2}{*}{ Gene } & \multirow[t]{2}{*}{ rs number } & \multirow[t]{2}{*}{ Risk allele } & \multicolumn{2}{|c|}{ Low level ${ }^{\mathrm{a}}$} & \multicolumn{2}{|c|}{ High level $^{\mathrm{a}}$} & \multirow[t]{2}{*}{$p_{\text {interaction }}$} \\
\hline & & & & $p^{\mathrm{b}}$ & OR $(95 \% \mathrm{CI})^{\mathrm{c}}$ & $p^{\mathrm{b}}$ & OR $(95 \% \mathrm{CI})^{\mathrm{c}}$ & \\
\hline \multirow[t]{6}{*}{ Genistein } & \multirow[t]{3}{*}{$A M D 1$} & rs1279599 & $\mathrm{T}$ & 0.21 & $1.94(0.81-4.64)$ & 0.39 & $1.42(0.59-3.43)$ & 0.77 \\
\hline & & rs7768897 & G & 0.17 & $1.98(0.83-4.70)$ & 0.39 & $1.42(0.59-3.43)$ & 0.74 \\
\hline & & rs811921 & $\mathrm{T}$ & 0.17 & $1.99(0.83-4.70)$ & 0.39 & $1.42(0.59-3.43)$ & 0.74 \\
\hline & OAZ2 & rs7403751 & $\mathrm{C}$ & 0.03 & $5.32(1.60-17.7)$ & 0.80 & $0.99(0.23-4.23)$ & 0.11 \\
\hline & \multirow[t]{2}{*}{$N Q O 1$} & rs1800566 & $\mathrm{T}$ & 0.19 & $1.53(0.57-4.11)$ & 0.03 & $0.36(0.15-0.90)$ & 0.04 \\
\hline & & rs1437135 & $\mathrm{G}$ & 0.19 & $1.53(0.57-4.11)$ & 0.03 & $0.36(0.15-0.90)$ & 0.04 \\
\hline \multirow[t]{6}{*}{ Daidzein } & \multirow[t]{3}{*}{$A M D 1$} & rs1279599 & $\mathrm{T}$ & 0.36 & $1.79(0.74-4.35)$ & 0.06 & $0.21(0.14-1.03)$ & 0.03 \\
\hline & & rs7768897 & G & 0.36 & $1.79(0.74-4.35)$ & 0.06 & $0.22(0.25-1.03)$ & $\mathbf{0 . 0 3}$ \\
\hline & & rs811921 & $\mathrm{T}$ & 0.36 & $1.79(0.74-4.35)$ & 0.06 & $0.22(0.25-1.03)$ & 0.03 \\
\hline & $O A Z 2$ & rs7403751 & $\mathrm{C}$ & 0.05 & $6.26(1.66-23.63)$ & 0.44 & $1.33(0.36-4.96)$ & 0.18 \\
\hline & \multirow[t]{2}{*}{$N Q O 1$} & rs1800566 & $\mathrm{T}$ & 0.06 & $2.28(0.82-6.30)$ & $<0.01$ & $0.26(0.10-0.64)$ & $<0.01$ \\
\hline & & rs1437135 & G & 0.06 & $2.28(0.82-6.30)$ & $<0.01$ & $0.26(0.10-0.64)$ & $<0.01$ \\
\hline \multirow[t]{6}{*}{ Equol } & \multirow[t]{3}{*}{$A M D 1$} & rs1279599 & $\mathrm{T}$ & 0.26 & $1.67(0.66-4.25)$ & 0.35 & $1.41(0.61-3.29)$ & 0.88 \\
\hline & & rs7768897 & G & 0.26 & $1.67(0.66-4.25)$ & 0.30 & $1.46(0.63-3.39)$ & 0.90 \\
\hline & & rs811921 & $\mathrm{T}$ & 0.26 & $1.67(0.66-4.25)$ & 0.30 & $1.46(0.63-3.39)$ & 0.90 \\
\hline & OAZ2 & rs7403751 & $\mathrm{C}$ & $<0.01$ & $6.49(1.78-23.70)$ & 1.00 & $0.92(0.21-3.97)$ & 0.04 \\
\hline & \multirow[t]{2}{*}{$N Q O 1$} & rs 1800566 & $\mathrm{~T}$ & 0.54 & $1.37(0.50-3.77)$ & 0.21 & $0.42(0.17-1.04)$ & 0.12 \\
\hline & & rs 1437135 & G & 0.54 & $1.37(0.50-3.77)$ & 0.21 & $0.42(0.17-1.04)$ & 0.12 \\
\hline \multirow[t]{6}{*}{ Enterolactone } & \multirow[t]{3}{*}{$A M D 1$} & rs 1279599 & $\mathrm{~T}$ & 0.01 & $3.13(1.29-7.61)$ & 0.68 & $0.85(0.35-2.08)$ & $\mathbf{0 . 0 3}$ \\
\hline & & rs7768897 & G & 0.01 & $3.22(1.33-7.80)$ & 0.68 & $0.85(0.35-2.08)$ & 0.03 \\
\hline & & rs811921 & $\mathrm{T}$ & 0.01 & $3.22(1.33-7.80)$ & 0.68 & $0.85(0.35-2.08)$ & 0.03 \\
\hline & $O A Z 2$ & rs7403751 & $\mathrm{C}$ & 0.40 & $2.06(0.59-7.17)$ & 0.06 & $0.24(0.07-0.89)$ & 0.57 \\
\hline & \multirow[t]{2}{*}{$N Q O 1$} & rs1800566 & $\mathrm{T}$ & 0.90 & $0.91(0.37-2.26)$ & 0.47 & $0.53(0.21-1.34)$ & 0.52 \\
\hline & & rs1437135 & G & 0.90 & $0.91(0.37-2.26)$ & 0.47 & $0.53(0.21-1.34)$ & 0.52 \\
\hline
\end{tabular}

Bold values indicate $p<0.05$

${ }^{\text {a }}$ According to the median blood phytoestrogen concentration among controls (low concentration, below the median; high concentration, the median or above). The median concentration of each phytoestrogen was as follows: $225.85 \mathrm{nmol} / \mathrm{L}$ for genistein, $134.20 \mathrm{nmol} / \mathrm{L}$ for daidzein, $37.19 \mathrm{nmol} / \mathrm{L}$ for equol, and $54.20 \mathrm{nmol} / \mathrm{L}$ for enterolactone.

b Wald $p$ value in the dominant model

c Adjusted for age, CagA seropositivity, H. pylori, and smoking status in the dominant model. 
Table 4 Pooled analysis (406 gastric cancer casess and 417 controls) for the interaction between selected SNPs of genes involved in the ODC pathway and phytoestrogen concentrations to modify gastric cancer risk in a nested case-control study within the Korean Multicenter Cancer Cohort

\begin{tabular}{|c|c|c|c|c|c|c|}
\hline Phytoestrogen & Gene & rs number & Risk allele & $\begin{array}{l}\text { Low level } \\
\text { OR }(95 \% \mathrm{CI})^{\mathrm{b}}\end{array}$ & $\begin{array}{l}\text { High level }^{\mathrm{a}} \\
\text { OR }(95 \% \mathrm{CI})^{\mathrm{c}}\end{array}$ & $p_{\text {interaction }}$ \\
\hline \multirow[t]{4}{*}{ Genistein } & $A M D 1$ & rs1279599 & $\mathrm{T}$ & $1.27(0.84-1.93)$ & $0.97(0.75-1.26)$ & 0.32 \\
\hline & & rs7768897 & G & $0.31(0.87-1.97)$ & $0.95(0.74-1.24)$ & 0.23 \\
\hline & $O A Z 2$ & rs 7403751 & $\mathrm{C}$ & $1.13(0.61-2.09)$ & $0.72(0.46-1.12)$ & 0.25 \\
\hline & $N Q O 1$ & rs1800566 & $\mathrm{T}$ & $1.46(0.98-2.19)$ & $0.68(0.53-0.88)$ & 0.002 \\
\hline \multirow[t]{4}{*}{ Daidzein } & $A M D 1$ & rs1279599 & $\mathrm{T}$ & $1.59(1.08-2.32)$ & $0.84(0.64-1.11)$ & 0.006 \\
\hline & & rs7768897 & G & $1.59(1.08-2.32)$ & $0.84(0.64-1.11)$ & 0.006 \\
\hline & OAZ2 & rs7403751 & $\mathrm{C}$ & $1.52(0.86-2.68)$ & $0.58(0.36-0.92)$ & 0.008 \\
\hline & $N Q O 1$ & rs 1800566 & $\mathrm{~T}$ & $1.19(0.83-1.72)$ & $0.73(0.56-0.96)$ & 0.005 \\
\hline
\end{tabular}

Merged data of genetic study results from discovery and extension phases and phytoestrogen experiments conducted in 2008 and 2011

Bold values indicate $p<0.05$

${ }^{a}$ The selected SNPs were also replicated with significant association with gastric cancer in the extension phase.

b According to the median blood phytoestrogen concentration among controls (low concentration, below the median; high concentration, the median or above). The median concentration of each phytoestrogen was as follows: $225.85 \mathrm{nmol} / \mathrm{L}$ for genistein, $134.20 \mathrm{nmol} / \mathrm{L}$ for daidzein, $37.19 \mathrm{nmol} / \mathrm{L}$ for equol, and $54.20 \mathrm{nmol} / \mathrm{L}$ for enterolactone

c Adjusted for age, CagA seropositivity, H. pylori, and smoking status in the dominant model

( $p_{\text {interaction }}=0.002$ and 0.005 , respectively). The gastric cancer risk of $N Q O 1$ rs1800566 changed according to the genistein and daidzein concentrations: the $\mathrm{T}$ allele of NQO1 rs1800566 was associated with a reduced risk of gastric cancer at high genistein and daidzein levels [OR 0.68 (95\% CI $0.53-0.88)$ and OR 0.73 (95\% CI 0.56-0.96), respectively], whereas it was nonsignificantly associated with an increased risk of gastric cancer at low genistein levels [OR 1.46 (95\% CI 0.98-2.19)] and was not associated with an increased risk of gastric cancer at low daidzein levels [OR 1.19 (95 \% CI 0.83-1.72)]. Major alleles of AMD1 rs1279599, AMD1 rs7768897, and OAZ2 rs7403751 had significant gene-daidzein interaction effects to modify gastric cancer risk ( $p_{\text {interaction }}=0.006,0.006$, and 0.008 , respectively). At high daidzein concentration, OAZ2 rs7403751 showed a significantly decreased gastric cancer risk [OR 0.58 (95\% CI 0.36-0.92)]. At low daidzein levels, both AMDI rs1279599 and AMDI rs7768897 had significant increased risks [both ORs 1.59 (95\% CIs 1.08-2.32)], whereas at high daidzein levels, no associations were observed [both ORs 0.84 (95\% CIs 0.64-1.11)] (Table 4).

\section{Discussion}

NQO1 rs1800566 showed a significant association with gastric cancer risk, and an interaction with genistein and daidzein could modify gastric cancer risk. Gene-environment analysis demonstrated significant interaction effects between genes controlling ODC such as NQOI,
$A M D 1$, and $O A Z 2$, and isoflavones such as genistein and daidzein.

The ODC-polyamine biosynthesis pathway is illustrated in Fig. 1. H. pylori stimulates ODC in host macrophages and arginase II. ODC metabolizes L-ornithine to polyamines (spermine, spermidine, putrescine). The presence of $H$. pylori induces macrophage iNOS for host immunity activation, whereas spermine inhibits expression of NOS2A (encoding iNOS). Then iNOS converts L-arginine into citrulline and $\mathrm{NO}$, where the latter possess antimicrobial and proinflammatory properties [12], leading to killing of H. pylori. Spermine to spermidine back-conversion by spermine oxidase, also induced by the presence of $H$. pylori [13], produces aldehyde (3-aminopropanal) and $\mathrm{H}_{2} \mathrm{O}_{2}$ [14]. Both by-products play a role in gastric carcinogenesis through DNA, RNA, protein, and membrane damage caused by reactive oxygen radicals [15]. Polyamine synthesis is stimulated by ODC and AMD [3, 4]. ODC is controlled by NQO1 and ornithine decarboxylase antizyme 1 (OAZ1), and polyamines stimulate OAZ1. NQO1 induces ODC by binding to and stabilizing ODC [16], whereas OAZ1 inactivates ODC by degradation [17]. The sequential processes in the ODC-polyamine pathway can be regulated by their host genes, thereby affecting cancer susceptibility by altering expression or function of enzymes to modulate polyamine synthesis.

NQO1 is a member of the $\mathrm{NAD}(\mathrm{P}) \mathrm{H}$ dehydrogenase (quinone) family that reduces quinones to hydroquinones, and participates in reducing the levels of free radicals. It binds to and stabilizes ODC, which attenuates the immune response through elevated levels of polyamines [16]. In this 
study, NQO1 rs1800566 alone showed an association with gastric cancer risk, and an interaction with genistein and daidzein modified gastric cancer risk according to isoflavone concentration. Previous studies support our results. Ikeda et al. [18] reported the T allele of NQO1 rs 1800566 was associated with a reduced risk of gastric cancer, although Hamajima et al. [19] did not find a statistical significance with gastric cancer risk. NQO1 rs1800566 is a functional SNP in NQO1 [C609T (Pro187Ser)]. The T allele has null enzyme activity, whereas the $\mathrm{C}$ allele has full enzyme activity [20], and the enzyme activity of the CT genotype decreases to approximately one third of that of the wild genotype (CC) [21, 22]. In our study, the T allele of NQO1 rs1800566 was associated with reduced risk of gastric cancer. On the basis of previous studies, the NQO1 T allele encodes the null NQO1 enzyme activity, which is reduced in the ODC pathway, and thus is involved in decreasing polyamine synthesis. The decrease in polyamine levels does not inhibit iNOS function to produce NO. Thus, the $N Q O 1 \mathrm{~T}$ allele could be indirectly related to less persistent $H$. pylori infection. Another study indirectly supports our results: Goto et al. [23] showed the CC genotype of the same $N Q O 1$ gene variant was associated with $H$. pylori seropositivity. Therefore, our result that the NQO1 rs1800566 T allele is associated with low risk of gastric cancer is biologically plausible.

Our study showed an interaction effect between $\mathrm{NQOI}$ rs1800566 and isoflavones (genistein and daidzein) to modify gastric cancer risk. An in vivo study supports our interaction result. Wiegand et al. [24] reported that genistein significantly increased activity levels of NQO1. Although the need for NQO1 at high isoflavone concentration was increased, the NQO1 rs $1800566 \mathrm{~T}$ allele encoded low activity of NQO1, and NQO1 encoded by the $\mathrm{T}$ allele reduces the function of $\mathrm{ODC}$, leading to decreased polyamine synthesis. Moreover, isoflavone is directly involved in decreasing ODC activity [7]. ODC inhibition by isoflavone alone and low NQO1 enzymatic activity caused by the rs $1800566 \mathrm{~T}$ allele can result in decreased polyamine synthesis. Therefore, interaction between $N Q O 1$ rs1800566 and isoflavone could modify gastric cancer risk.

Other mechanisms to control polyamine synthesis are by $O A Z 1$ and AMD1. Although it is not known whether isoflavones control OAZ1 and AMD1, it has been hypothesized that if isoflavones stimulate OAZ1, and the OAZ1 C allele is a high-activity allele, the amount of highly active OAZ1 increases, leading to degradation of ODC and decreased polyamine synthesis. In contrast, if isoflavones induce AMD1 and the major allele of $A M D 1$ is related to low enzyme activity, the low-activity AMD1 could decrease polyamine synthesis. Future investigations should assess whether the interaction with isoflavones observed for gastric cancer could be explained by biological interaction between AMD1 and OAZ1 and isoflavones and should study functional $A M D 1$ and $O A Z 1$ SNPs.

Limitations should be noted. In the extension phase, hospital and community-based casess were matched with community-based controls, which may introduce bias. However, information bias is minimized since people are born with their genes and changes in genes are not common. Although this was a two-phase study that aimed to increase the power of the study, we were not able to stratify the study with regard to important factors such as type (cardiac and noncardiac) and variants (intestinal and diffuse). Also, selection bias was minimized because cases were matched with controls according to important risk factors in the initial study design stage.

The study is a two-phase population-based nested casecontrol genetic association study that is free of many biases common in retrospective designs. Confounding factors were adjusted for in the multivariate models. In the geneenvironment analysis, phytoestrogen biomarkers were measured from blood samples, which are less subject to recall bias compared with food frequency questionnaires.

In conclusion, we report that $N Q O 1$ rs 1800566 involved in the ODC pathway can be a genetic susceptibility factor for gastric cancer. Genes involved in the ODC pathway (NQO1, AMDI, and OAZ) and isoflavones (genistein and daidzein) showed a significant interaction to modify gastric cancer risk. Replication and validation genetic studies with a greater number of subjects conducted in other ethnic populations in addition to in-depth in vitro and animal studies on the real metabolic function and cellular events of the gene variants in gastric cancer tissue will elucidate and further clarify the biological pathways, causal mechanisms, and genetic and environmental individual and interactive effects involved in gastric carcinogenesis.

Acknowledgments This study was supported by a grants from the National R\&D Program for Cancer Control, Ministry of Health \& Welfare, Republic of Korea (0520140), the Basic Science Research Program through the National Research Foundation of Korea funded by the Ministry of Education, Science and Technology (KRF-2007313-E00175), and the Korean Foundation for Cancer Research (KFCR-CB-2013-01).

Conflict of interest The authors declare no competing interests.

\section{References}

1. International Agency for Research on Cancer. IARC monographs on the evaluation of carcinogenic risks to humans. Vol 61., Schistosomes, liver flukes and Helicobacter pylori. Lyon: International Agency for Research on Cancer; 1994; p. 61.

2. Pricci M, Linsalata M, Russo F, Messa C, Amati L, Caradonna L, Jirillo E, Di Leo A. Effects of $17 \beta$-estradiol administration on apoptosis and polyamine content in AGS cell line. Anticancer Res. 2001;21(5):3215-20. 
3. Wada M, Shirahata A. Conformational stabilization of rat $s$ adenosylmethionine decarboxylase by putrescine. Biol Pharm Bull. 2010;33(11):1800-5.

4. Pegg AE. S-Adenosylmethionine decarboxylase. Essays Biochem. 2009;46:25-45.

5. O'Brien TG, Megosh LC, Gilliard G, Soler AP. Ornithine decarboxylase overexpression is a sufficient condition for tumor promotion in mouse skin. Cancer Res. 1997;57(13):2630-7.

6. Megosh LC, Hu J, George K, O'Brien TG. Genetic control of polyamine-dependent susceptibility to skin tumorigenesis. Genomics. 2002;79(4):505-12.

7. Linsalata M, Russo F, Notarnicola M, Guerra V, Cavallini A, Clemente C, Messa C. Effects of genistein on the polyamine metabolism and cell growth in DLD-1 human colon cancer cells. Nutr Cancer. 2005;52(1):84-93.

8. Yoo KY, Shin HR, Chang SH, Lee KS, Park SK, Kang D, Lee DH. Korean multi-center cancer cohort study including a biological materials bank (KMCC-I). Asian Pac J Cancer Prev. 2002;3(1):85-92.

9. Cho LY, Kim CS, Li L, Yang JJ, Park B, Shin A, Chang SH, Lee KS, Kim H, Yoo KY, et al. Validation of self-reported cancer incidence at follow-up in a prospective cohort study. Ann Epidemiol. 2009;19(9):644-6.

10. Ko KP, Park SK, Park B, Yang JJ, Cho LY, Kang C, Kim CS, Gwack J, Shin A, Kim Y, et al. Isoflavones from phytoestrogens and gastric cancer risk: a nested case-control study within the Korean Multicenter Cancer Cohort. Cancer Epidemiol Biomarkers Prev. 2010;19(5):1292-300.

11. Purcell S, Neale B, Todd-Brown K, Thomas L, Ferreira MA, Bender D, Maller J, Sklar P, de Bakker PI, Daly MJ, et al. PLINK: a tool set for whole-genome association and populationbased linkage analyses. Am J Hum Genet. 2007;81(3):559-75.

12. Chaturvedi R, Asim M, Hoge S, Lewis ND, Singh K, Barry DP, de Sablet T, Piazuelo MB, Sarvaria AR, Cheng Y, et al. Polyamines impair immunity to Helicobacter pylori by inhibiting Larginine uptake required for nitric oxide production. Gastroenterology. 2010;139(5):1686-98.

13. Chaturvedi R, Cheng Y, Asim M, Bussiere FI, Xu H, Gobert AP, Hacker A, Casero RA Jr, Wilson KT. Induction of polyamine oxidase 1 by Helicobacter pylori causes macrophage apoptosis by hydrogen peroxide release and mitochondrial membrane depolarization. J Biol Chem. 2004;279(38):40161-73.
14. Gobert AP, Chaturvedi R, Wilson KT. Methods to evaluate alterations in polyamine metabolism caused by Helicobacter pylori infection. Methods Mol Biol. 2011;720:409-25.

15. Casero RA, Pegg AE. Polyamine catabolism and disease. Biochem J. 2009;421(3):323-38.

16. Asher G, Bercovich Z, Tsvetkov P, Shaul Y, Kahana C. $20 \mathrm{~S}$ proteasomal degradation of ornithine decarboxylase is regulated by NQO1. Mol Cell. 2005;17(5):645-55.

17. Kahana C. Identification, assay, and functional analysis of the antizyme inhibitor family. Methods Mol Biol. 2011;720:269-78.

18. Ikeda S, Sasazuki S, Natsukawa S, Shaura K, Koizumi Y, Kasuga Y, Ohnami S, Sakamoto H, Yoshida T, Iwasaki M, et al. Screening of 214 single nucleotide polymorphisms in 44 candidate cancer susceptibility genes: a case-control study on gastric and colorectal cancers in the Japanese population. Am J Gastroenterol. 2008;103(6):1476-87.

19. Hamajima N, Naito M, Kondo T, Goto Y. Genetic factors involved in the development of Helicobacter pylori-related gastric cancer. Cancer Sci. 2006;97(11):1129-38.

20. Siegel D, McGuinness SM, Winski SL, Ross D. Genotype-phenotype relationships in studies of a polymorphism in NAD(P)H:quinone oxidoreductase 1. Pharmacogenetics. 1999;9(1):113-21.

21. Kuehl BL, Paterson JW, Peacock JW, Paterson MC, Rauth AM. Presence of a heterozygous substitution and its relationship to DT-diaphorase activity. Br J Cancer. 1995;72(3):555-61.

22. Larson RA, Wang Y, Banerjee M, Wiemels J, Hartford C, Le Beau MM, Smith MT. Prevalence of the inactivating 609C $\rightarrow$ T polymorphism in the $\mathrm{NAD}(\mathrm{P}) \mathrm{H}$ :quinone oxidoreductase (NQO1) gene in patients with primary and therapy-related myeloid leukemia. Blood. 1999;94(2):803-7.

23. Goto Y, Hamajima N, Honda H, Matsuo K, Yamamoto K, Tamakoshi A, Ando T, Goto H. Association between Helicobacter pylori seropositivity and $\mathrm{NAD}(\mathrm{P}) \mathrm{H}$ :quinone oxidoreductase 1 (NQO1) C609T polymorphism observed in outpatients and health checkup examinees. Gastric Cancer. 2005;8(1):12-7.

24. Wiegand H, Wagner AE, Boesch-Saadatmandi C, Kruse HP, Kulling S, Rimbach G. Effect of dietary genistein on phase II and antioxidant enzymes in rat liver. Cancer Genomics Proteomics. 2009;6(2):85-92. 